\title{
Konik işınlı bilgisayarlı tomografide maksiller ve mandibular süpernümere dişlerin lokalizasyonları, karakteristikleri ve komplikasyonları
}

Dilek Menziletoğlu(0000-0002-2539-0688) ${ }^{\alpha}$, Emire Aybüke Erdur(0000-0002-1887-8474) ${ }^{\beta}$, Funda Baştürk(0000-0002-7908-0929) ${ }^{\alpha}$

Selcuk Dent J, 2019; 6: 38-43 (Doi: 10.15311/selcukdentj.345366)

Bašvuru Tarihi: 20 Ekim 2017 Yayına Kabul Tarihi: 26 Kasım 2018

\section{öz}

Konik işınlı bilgisayarlı tomografide maksiller ve mandibular süpernümere dişlerin lokalizasyonları, karakteristikleri ve komplikasyonları

Amaç: Süpernümere dişler, normal diş sayısından fazla olan dişler olarak tanımlanmaktadır. Hem süt hemde daimi dentisyonda süpernümere dişlere rastlanılmaktadır. Etiyolojileri tam olarak bilinmemektedir. Genelde gömülüdür ve radyograflarda tesadüfen görülürler. Bu çalışmanın amacı, süpernümere dişlerin panoramik radyograf ve konik ışınlı bilgisayarlı tomografi (KIBT) kullanılarak lokalizasyonunu değerlendirmek ve tedavi yaklaşımlarını sunmaktadır.

Gereç ve Yöntemler: Bu retrospektif çalışmada 6273 hastanın radyografik verileri Necmettin Erbakan Üniversitesi, Diş Hekimliği Fakültesi Ağız, Diş Çene Cerrahisi ve Ortodonti bölümlerinde incelendi. Süpernümere dişlerin sayısı, dişin anatomik lokalizasyonu, erüpsiyon durumu panoramik radyografta ve KIBT'ta incelendi.

Bulgular: 6273 hastanın 64'ünde (40 erkek, 24 kadın) 66 süpernümere diş teşhis edildi. Hastaların yaş aralığı 9-65 yıl ve yaş ortalaması 22.47 yıldı. İki hastada (mandibula premolar ve molar bölge) çift taraflı süpernümere diş vardı. Kırk üç süpernümere diş gömülüydü. Süpernümere dişlerin hepsi çekildi. 44 süpernümere diş ortodontik tedavi için çekildi. 2 hastada maksilla premolar bölgede görülen süpernümere diş ile birlikte görülen kist enükle edildi ve dişler çekildi. 14 süpernümere diş profilaktik olarak çekildi.

Sonuç: Süpernümere dişlerin büyük çoğunluğu gömülü olduğundan rutin klinik muayeneler sırasında teşhis edilememekte ancak radyografik incelemeler sırasında veya komplikasyonlara neden olduklarında saptanabilmektedir. Ortodontik tedavi gören hastalarda süpernümere dişler daimi dişlerin hareketine engel olmaktadır. Erken tanı ve tedavi komplikasyonların önlenmesi açısından önemlidir.

\section{ANAHTAR KELIMELER}

Konik ışınlı bilgisayarlı tomografi, süpernümere dişler, ortodontik tedavi

Süpernümere diş (SD); dentisyonda normal olması gereken diş sayısından fazla olan dişlerdir. Hem süt dentisyonunda, hem de daimi dentisyonda görülebileceği gibi bir veya birden fazla sayıda, unilateral veya bilateral olarak da bulunabilirler. ${ }^{1-4} \mathrm{Bu}$ dişlerin etiyolojilerinde genetik veya çevresel

\section{ABSTRACT}

Localization, characteristics and complications of the maxillary and mandibular supernumerary teeth in cone beam computed tomography

Background: Supernumerary teeth are described as the teeth formed in excess of the normal dental formula. They have been reported in both the primary and permanent dentition. Their etiology is still not clearly understood. They usually don't erupt and are seen incidentally on radiographies. The aim of study is to evaluate the localization of supernumerary teeth using panoramic radiography and conic beam computed tomography (CBCT) and to present treatment approaches.

Methods: In this retrospective study, radiographic data of 6273 patients were examined at Necmettin Erbakan University Faculty of Dentistry, department of oral and maxillofacial surgery and orthodontics. Number of supernumerary teeth patients tooth number, anatomical localization of the tooth, and status of the eruption were examined in CBCT and panoramic radiography.

Results: 66 supernumerary teeth were diagnosed in 64 (40 male, 24 female) of 6273 patients. The mean age of patients was 22.47 years (9-65years). Two patients have bilateral supernumerary teeth in premolar and molar region in mandible. 43 supernumerary teeth were impacted. All of the supernumerary teeth were extracted. 44 supernumerary teeth were extracted for the orthodontic treatment. There were two supernumerary teeth with cysts in two patients. 14 supernumerary teeth were extracted for profilactic indication.

Conclusion: Supernumerary teeth can't be diagnosed during routine clinical examination, because the majority of them are impacted. However they can only be detected during radiological examination or when they cause complications. The supernumerary teeth prevent the movement of permanent teeth in orthodontics patients. Early diagnosis and treatment are important to prevent the complications.

\section{KEYWORDS}

Cone-beam computed tomography, supernumerary teeth, orthodontic treatment

faktörlerin etkili olabileceği düşünülmesine rağmen etiyolojileri tam olarak bilinmemektedir. ${ }^{5}$ Dişlerin çene kemiği içindeki gelişimi sırasında dental laminadaki hiperaktiviteden dolayı süpernümere dişlerin oluşabileceği en çok kabul edilen hipotezdir. ${ }^{6}$

\footnotetext{
${ }^{\alpha}$ Necmettin Erbakan Üniversitesi, Diş Hekimliği Fakültesi, Ağız Diş ve Çene Cerrahisi Anabilim Dalı, Konya

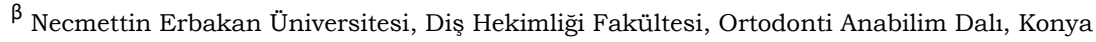


Genellikle asemptomatik olan SD'ler; kök rezorpsiyonu, yer değiştirme, komşu dişlerde çapraşıklık veya diestema, daimi dişlerin sürmesinde gecikme veya kist gibi komplikasyonlar oluşturabileceği bildirilmiştir.,3 SD'ler; Gardner Sendromu, dudak-damak yarığı, cleidocranial dysostosis ve orofasio-dijital sendrom gibi vakalarda oldukça sık görülmektedir. ${ }^{8}$ SD'nin doğru lokalizasyonu; teşhis, tedavi planı ve dişlerin çekiminde oldukça önemlidir. Geleneksel olarak SD; panoramik, oklüzal veya sefalometrik filmlerde teşhis edilir. ${ }^{3,7}$ İki boyutlu radyografik görüntüleme yöntemleri kullanılarak; dişin üç boyutlu konumunu belirlemek, komşu yapılar ve dişlerle olan ilişkisini saptamak mümkün değildir. SD'nin konumunun belirlenmesinde konik ışınlı bilgisayarlı tomografinin (KIBT) kullanımı ile iki boyutlu görüntülemenin eksikliğinin giderilebileceği savunulmaktadır. ${ }^{9}$

$\mathrm{Bu}$ çalışmanın amacı, SD'nin panoramik filmde ve KIBT'ta karakteristiklerini ve lokalizasyonlarını inceleyip, görülme sıklığını değerlendirmek ve komşu anatomik yapılarda herhangi bir komplikasyona neden olup olmadığını incelemektir.

\section{GEREÇ VE YÖNTEM}

Bu retrospektif çalışmada, Ocak 2016-Temmuz 2017tarihleri arasında dental problemlerden dolayı Necmettin Erbakan Üniversitesi, Diş Hekimliği Fakültesi Ağız, Diş Çene Cerrahisi ve Ortodonti bölümlerine başvuran 6273 hastanın panoramik filmleri incelendi. SD'si bulunan hastaların KIBT görüntüleri de incelenerek SD'nin sayısı, dişin anatomik lokalizasyonu, erüpsiyon durumu, neden olduğu patoloji ve yapılan tedaviler kayıt edildi. Elde edilen veriler tanımlayıcı istatistik yöntemi ile değerlendirildi.

\section{BULGULAR}

Çalışmaya dahil edilen 6273 hastanın 64'ünde (40 erkek, 24 kadın) 66 SD teşhis edildi. Hastaların yaş ortalaması 22.47 yıldı (Yaş aralığı 9-65 yıl) (Tablo 1). Hastaların 2975'i süt dentisyon döneminde, 3298'i ise daimi dentisyon dönemindeydi. Popülasyonda SD görülme sıklığı \%1.02 olarak bulundu.

Hastaların panoramik filmleri ve KIBT'ları incelendiğinde, 2 hastada (mandibula premolar ve molar bölge) çift taraflı SD mevcuttu. SD'nin \%75.76'sı maksillada, \%24.24'ü de mandibulada yer almaktaydı. 43 SD gömülü ve 23 'ü de sürmüş durumdaydı. 33 SD konik formda, 10'u odontoma ve 23'ü ise diş şeklindeydi. 40 SD eğimli, 5'i transvers, 1 'i ters pozisyonda ve 20'si de normal pozisyondaydı (Tablo 2). 44 SD ortodontik tedavi için çekildi. (Resim 1, Resim 2, Resim 3). 4 hastanın anterior bölgedeki dişlerine dolgu yapılacağından ve
2 hastanın da anterior bölgedeki dişlerine köprü yapılacağından dolayı 6 SD'nin bu tedavilere engel olacağı düşünülerek çekildi. 14 hastanın da rutin muayene sırasında görülen SD'leri profilaktik olarak çekildi. 2 hastanın maksillapremolar bölgesinde SD ile birlikte görülen odontojenik kist teşhis edildi (Resim 4, Resim 5). SD'nin çekimi ile birlikte kist enükle edildi. Patolojik inceleme sonucunda dentigeröz kist tanısı konuldu. Bu iki hastada SD'lerden kaynaklanan patolojik problem gözlenirken, geri kalan 62 hastada SD'lerin komşuluğunda olan dişlerde herhangi bir rezorpsiyon veya patolojik bir bulguya rastlanılmadı.

Tablo 1.

\section{Cinsiyete göre süpernümere diş sayısı ve insidansı}

\begin{tabular}{|lccc|}
\hline Cinsiyet & Popülasyon & $\begin{array}{c}\text { Süpernümere } \\
\text { dişi bulunan } \\
\text { hasta sayısı }\end{array}$ & Insidansı \\
\hline Kadın & 2972 & 24 & 0.81 \\
\hline Erkek & 3301 & 40 & 44197 \\
\hline Toplam & 6273 & 64 & 43497 \\
\hline
\end{tabular}

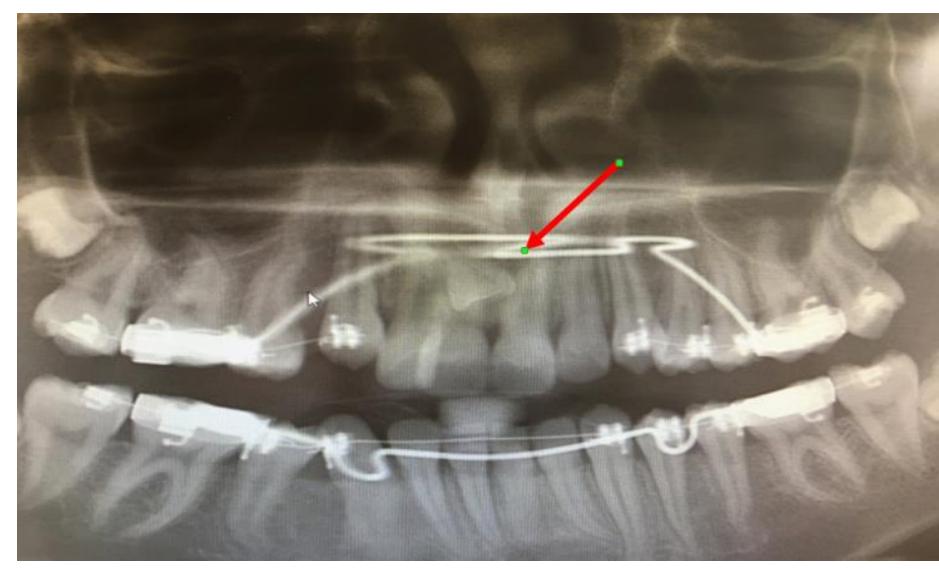

Resim 1.

Maksilla anterior bölgedeki süpernümere dişin panoramik radyograf görüntüsü

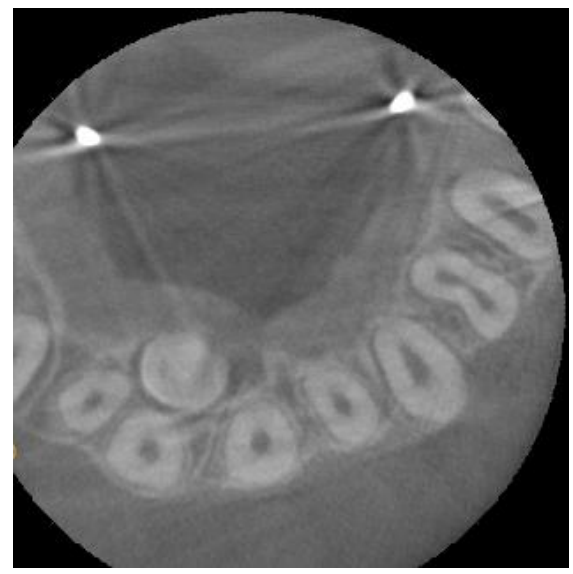

Resim 2.

Maksilla anterior bölgedeki süpernümere

dişin aksiyal kesitli KIBT görüntüsü 


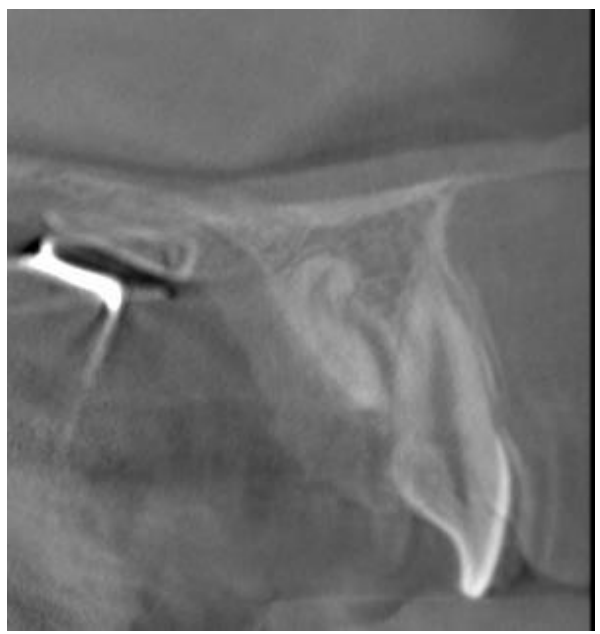

Resim 3.

Maksilla anterior bölgedeki süpernümere dişin sagittal kesitli KIBT görüntüsü

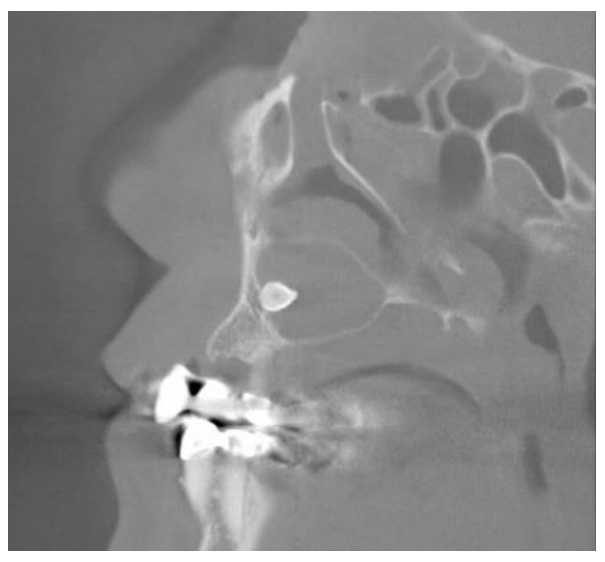

Resim 5.

Kist ile ilişkili süpernümere dişin sagittal kesitli KIBT görüntüsü

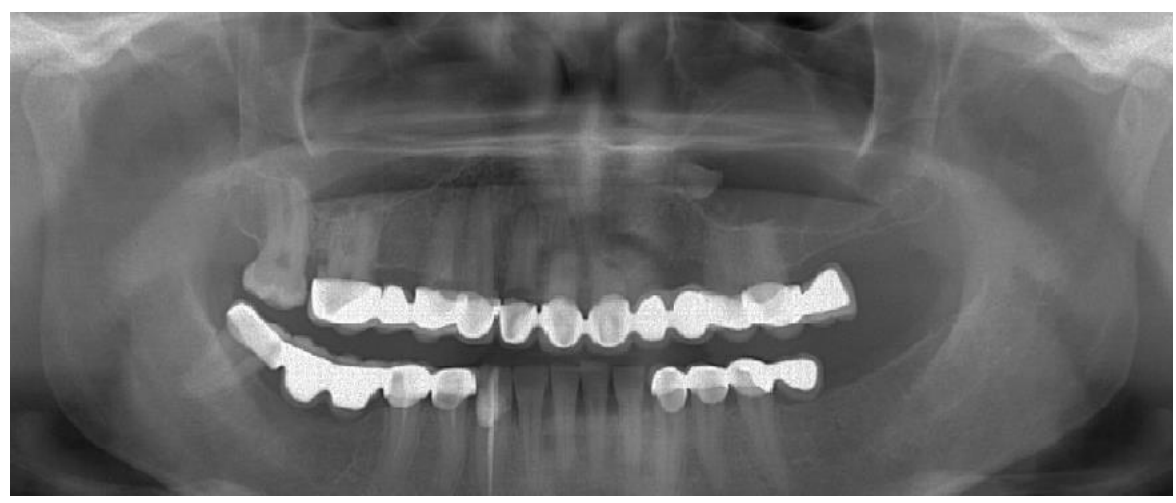

Resim 4.

Kist ile ilişkili süpernümere dişin panoramik görüntüsü

Tablo 2.

\section{Cinsiyete göre süpernümere diş sayısı ve insidansı}

\begin{tabular}{|llccc|}
\hline & & Anterior & Premolar & Molar \\
\hline \multirow{2}{*}{ Lokalizasyon } & Maksilla & 41 & 6 & 3 \\
\cline { 2 - 5 } & Mandibula & 3 & 10 & 3 \\
\hline \multirow{3}{*}{$\begin{array}{l}\text { Süpernümere dişin } \\
\text { şekli }\end{array}$} & Konik & 27 & 6 & - \\
\cline { 2 - 5 } & Odontoma & 7 & 3 & - \\
\cline { 2 - 5 } & Diş şeklinde & 13 & 6 & 4 \\
\hline \multirow{2}{*}{$\begin{array}{l}\text { Süpernümere dişin } \\
\text { sürme durumu }\end{array}$} & Gömülü & 26 & 12 & 5 \\
\cline { 2 - 5 } & Sürmüş & 19 & 4 & - \\
\hline \multirow{3}{*}{$\begin{array}{l}\text { Süpernümere dişin } \\
\text { pozisyonu }\end{array}$} & Eğimli & 30 & 7 & 3 \\
\cline { 2 - 5 } & Ters pozisyon & 1 & - & - \\
\cline { 2 - 5 } & Normal pozisyon & 13 & 7 & - \\
\cline { 2 - 5 } & Transvers & 2 & 2 & 1 \\
\hline
\end{tabular}

\section{TARTIŞMA}

Daimi dentisyonda ve süt dentisyonda SD'lerin görüldüğü ancak süt dentisyonunda görülme sıklığının daha az olduğu ve bu oranın \%0.3-0.6 arasında değiştiği bildirilmiştir. Genel olarak, SD'nin popülasyondaki insidansı \%0.1-3.8 arasında değişmektedir. ${ }^{1,10-13}$ Çalışmamızda, 66 SD'in 26'sı süt dentisyonda, 40'ı ise daimi dentisyonda gözlendi. SD'lerin görülme sıklı̆ı \% 1.02 ve süt dentisyondaki ise $\% 0.80^{\prime} \mathrm{di}$.

Esenlik ve ark. ${ }^{10}$ yapmış oldukları 2599 hastanın incelendiği bir çalışmada, 69 hastada SD'in mevcut olduğunu ve yaşlarının ise 6-16 yıl arasında değiştiğini bildirmişlerdir. Küchler ve ark. ${ }^{13}$, SD'nin görülme sıklığının \%2.3 olduğunu ve popülasyonun yaş aralığının 6-12 yıl arasında değiştiğini belirtmişlerdir. Bu çalışmaların aksine, çalışmamızda yaş aralığımı 9-65 yıl arasında değişmektedir. Hem süt hem de daimi dentisyonda SD'ye rastlanılmıştır.

Literatürde, SD'nin erkeklerde görülme sıklığının kadınlara oranla daha yüksek olduğu vurgulanmaktadır. ${ }^{1,14,15}$ Bizim bulgularımızda erkeklerde SD kadınlardan daha çok görülmekte ve literatürle uyumlu sonuçlar vermektedir.

Çenelerin herhangi bir bölgesinde SD görülebilir fakat en fazla anterior maksillada görüldüğü belirtilmiştir. 1,10,16 Sharma ve ark. ${ }^{17}$ yapmış oldukları çalışmada, SD'nin \%81.2'sinin santral kesici bölgesinde lokalize olduğunu bildirmişlerdir. Hastalarımızda görülen SD'nin en çok 
maksilla ve mandibulanın anterior bölgesinde (\%66.66) yer aldığı gözlendi. Bu durumun aksine en az ise molar bölgede SD mevcuttu.

Bir bireyin alt veya üst çenesinin herhangi bir bölgesinde tek veya birden fazla SD gözlenebilir. ${ }^{3}$ Daha sıklıkla tek olarak bulunurken, birden fazla olduğu durumlarda da iki SD'nin bulunması daha yaygındır. 1,3,10,17 Nadirde olsa bazı hastalıklarla veya sendromlarla birlikte de SD bulunabilir. ${ }^{18}$ Bizim sonuçlarımıza göre, 62 SD alt veya üst çenenin herhangi bir bölgesinde ve tekti. Sadece iki hastada çift taraflı SD bulunmaktaydı.

Rajab ve ark3, SD'yi; konik, tüberküllü, odontoma ve normal diş formu olmak üzere morfolojilerine göre sınıflandırmışlardır. Gülhan ${ }^{19}$, SD'nin en çok konik şekilli olarak görüldüğünü bildirmiştir. Bizim araştırmamızda literatürle uyumlu olarak, 64 hastanın 33 'ünde da konik formda SD mevcuttu. En fazla konik formlu SD gözlenirken, diş şeklinde görülenlerin sayısı 23 ve odontoma şeklinde olanların sayısı ise 10 olarak bulundu.

Literatürde, SD'si olan hastalarda KIBT'ın kullanımı bildirilmiştir. Panoramik veya periapikal filmler kullanıldığında karşılaşılan magnifikasyon, distorsiyon veya görüntülerin süperpose olması gibi problemler KIBT görüntüleri ile azaltılmıştır. ${ }^{20}$ SD'in; özellikle nasal taban, nasopalatin kanal veya ağız tabanı gibi önemli anatomik yapılara yakınlığı varsa, cerrahi öncesi ve bölümler arası yapılacak müdahalede 3 boyutlu doğru teşhis intiyacı ortaya çıkmaktadır. Son zamanlarda yapılan çalışmalarda geleneksel radyografla KIBT karşılaştırıldığında 3boyutlu görüntülemenin kök rezorpsiyonunun varlığı ve şiddeti hakkında daha çok bilgi verdiği belirtilmektedir. ${ }^{20-22}$ Çene cerrahları KIBT görüntüleri sayesinde özellikle sürmemiş SD'leri çekerken vestibülden mi yoksa palatinal veya lingualden mi dişe ulaşacaklarına ve diğer dişlerin kökleri ile önemli anatomik yapılara zarar vermeden dişleri nasıl çekeceklerini planlarlar. Böylelikle oluşabilecek postoperatif komplikasyonların sayısı da azalacaktır.

Merrett ve ark. ${ }^{23} \mathrm{KBIT}$ 'ın SD'li hastaların tedavi planlamasında oldukça yararlı bir alet olduğunu vurgulamışlardır. Mossaz ve ark. ${ }^{24}$ KIBT ile SD'lerin morfolojik karakteristiklerinin rahatlıkla incelenebileceğini, komşu dişlerin köklerinde herhangi bir rezorpsiyon olup olmadığının net bir şekilde görülebileceğini bildirmişlerdir. Raupp ve ark. ${ }^{25}$ yapmış oldukları çalışmada, bilgisayarlı tomografinin SD'lerin lokalizasyonunun belirlenmesinde etkili olduğunu, cerrahi tedavinin planlanmasında kesin bilgi verdiğini ve operasyonun süresini azalttığını belirtmişlerdir.
SD'nin; eğer komşu diş kökünde rezorpsiyona, daimi dişin sưrmesinde gecikme veya yer değştirmesi gibi komplikasyonlara neden olabileceğ şüphesi varsa veya komplikasyona neden oluşmuşsa bu dişler genelde çekilir. SD'nin erken çekilmesinin bu dişlerle ilişkide bulunan komşu dişlerin etkilenme intimalini ve sürme potansiyelindeki kaybı azaltacağ, yer kaybı veya orta hattaki kaymalara engel olacagı ve bu sayede daha ileri cerrahi veya ortodontik tedavi gerekliligini ortadan kaldıracağ daha önceki çalışmalarda belirtilmiştir. ${ }^{26}$ Çalışmamızda, ortodontik tedavi görecek 42 hastanın SD'si gecikmiş sürme veya çapraşıklık nedeni ile çekildi. Sadece 2 hastada SD'nin etrafında odontojenik kist görüldü. Hastaların hepsinden KIBT alındığı için kistin boyutu ve lokalizasyonuda net bir şekilde görüldü. Kist enükle edildi ve patolojik tanısı dentigeröz kist olarak konuldu. Ertaş ve ark. ${ }^{14}$ ile Tatlı ve ark. ${ }^{27}$ yapmış oldukları çalışmalarla uyumlu olarak, komşu dişlerde SD'nin neden olduğu herhangi bir kök rezorpsiyonu gözlenmedi.

\section{SONUÇ}

Süpernümere dişler sıklıkla gömülüdür ve rutin klinik muayenede gözden kaçabilirler. Çoğunlukla radyografik muayenede teşhis edilirler. Panoramik, periapikal veya oklüzal filmler SD'lerin lokalizasyonunu tam olarak belirlemede yetersiz kalmaktadır. Alınan KIBT ile SD'nin lokalizasyonu, o bölgede meydana gelen patolojik bir durum veya komşu dişlerde oluşan rezorpsiyon net bir şekilde görülmektedir. KIBT görüntüleri, cerrahi müdahalenin planlanmasında ve uygulanmasında kolaylık sağlamaktadır. SD'lerin teşhisindeki gecikme yapılacak olan tedavilerin daha kapsamlı olmasına ve bazı komplikasyonlara neden olabilir. Erken dönemde yapılan teşhisler, dental anomaliler ile ilişkili ortodontik problemleri veya patolojilerin oluşmasını önleyecek ve yapılacak tedavilerin kapsamı büyümeden müdahale edilmiş olacaktır. 


\section{KAYNAKLAR}

1. Celikoglu M, Kamak H, Oktay $H$. Prevalence andcharacteristics of supernumerary teeth in a nonsyndromeTurkish population: associated pathologies and proposedtreatment. Med Oral Patol Oral Cir Bucal 2010; 15: 575-8.

2. Fardi A, Kondylidou-Sidira A, Bachour Z, Parisis N, TsirlisA. Incidence of impacted and supernumerary teeth-aradiographic study in a North Greek population. Med Oral Patol Oral Cir Bucal 2011; 16: 56-61.

3. Rajab L, Hamdan M. Supernumerary teeth: review of theliterature and a survey of 152 cases. Int J Paediatr Dent 2002; 12: 244-54.

4. Pereira MN, de Almeida LE, Martins MT, da Silva CamposMJ, Fraga MR, Vitral RW. Multiple hyperdontia: Reportof an unusual case. Am J Orthod Dentofacial Orthop 2011; 140: 580-4.

5. Primosch RE. Anterior supernumerary teethassessment and surgical intervention in children. Pediatr Dent 1981; 3: 204-15.

6. Nasif MW, Ruffalo RC, Zullo T. Impacted supernumerary teeth: a survey of 50 cases. J Am Dent Assoc 1983; 106: 201-4.

7. Garvey T, Barry H J, Blake M. Supernumerary teeth an overview of classification, diagnosis and management. J Can Dent Assoc 1999; 65: 612-6.

8. Moore SR, Wilson DF, Kibble J. Sequential development of multiple supernumerary teeth in the mandibular premolar region-a radiographic case report. Int J Paediatr Dent 2002; 12: 143-5.

9. Kapila S, Conley RS, Harrell WEJ. The current status of cone beam computed tomography imaging in orthodontics. Dentomaxillofac Radiol 2011; 40: 24 34.

10. Esenlik E, Sayin MO, Atilla AO, Ozen T, Altun C, Başak $F$. Supernumerary teeth in a Turkish population. Am J Orthod Dentofacial Orthop 2009; 136: 848-52.

11. Martínez-González JM, Cortés-Bretón Brinkmann J, Calvo-Guirado JL, Arias Irimia O, Barona-Dorado C. Clinical epidemiological analysis of 173 supernumerary molars. Acta Odontol Scand 2012; 70: 398-404.

12. Kumar DK, Gopal KS. An epidemiological study on supernumerary teeth: A survey on 5,000 people. J Clin Diagn Res 2013; 7: 1504-7.

13. Küchler EC, Costa AG, Costa Mde C, Vieira AR, Granjeiro JM. Supernumerary teeth vary depending on gender. Braz Oral Res 2011; 25: 76-9.

14. Ertaş ET, Şişman Y, Yırcalı MA. Bir grup Türk popülasyonunda görülen meziodenslerin sıklığı, özellikleri ve meydana getirdikleri komplikasyonlar. Cumhuriyet Dent J 2013; 16: 267-2.
15. Harris EF, Clark LL. An epidemiological study of hyperdontia in American blacks and whites. Angle Orthod 2008; 78: 460-5.

16. De Oliveira Gomes C, Drummond SN, Jham BC, Abdo EN, Mesquita RA. A survey of 460 supernumerary teeth in Brazilian children and adolescents. Int J Paediatr Dent 2008; 18: 98106.

17. Sharma A, Singh VP. Supernumerary teeth in Indian children: A survey of 300 cases. Int J Dent 2012; 2012: 745265.

18. Açikgöz A, Açikgöz G, Tunga U, Otan F. Characteristics and prevalence of non-syndrome multiple supernumerary teeth: $A$ retrospective study. Dentomaxillofac Radiol 2006; 35: 185-90.

19.Gülhan, A. Pedodonti. Istanbul: Doyuran MatbaasI; 1987; 131-5.

20.Botticelli S, Verna C, Cattaneo PM, Heidmann J, Melsen B. Two- versus three-dimensional imaging in subjects with unerupted maxillary canines. Eur J Orthod 2011; 33: 344-9.

21. Katheria BC, Kau CH, Tate R, Chen JW, English $\mathrm{J}$, Bouquot J. Effectiveness of impacted and supernumerary tooth diagnosis from traditional radiography versus cone beam computed tomographyCone-beam computed tomography of the maxillofacial region-an update. A novel 3D classification system for canine impactions-the KPG index. Pediatr Dent 2010; 32: 304-9.

22. Alqerban $A$, Jacobs $R$, Fieuws $S$, Nackaerts $O$, The Sedentexct Project Consortium, Willems G. Comparison of 6 cone-beam computed tomography systems for image quality and detection of simulated canine impaction-induced external root resorption in maxillary lateral incisors. Am J Orthod Dentofacial Orthop. 2011; 140: 29-39.

23. Merrett SJ, Drage N, Siphahi D. The use of cone beam computed tomography in planning supernumerary cases. J Orthodontics 2013; 40: 38-46.

24.Mossaz J, Kloukos D, Pandis N, Suter GAV, Katsaros C, Bornstein MM. Morphologic characteristics, location and associated complications of maxillary and mandibular supernumerary teeth as evaluated using cone beam computed tomography. European $\mathrm{J}$ Orthodontics 2014: 36; 708-18.

25.Raupp S, Kramer PF, de Oliveira HW, da Rosa FM, Faraco IMJr. Application of computed tomograpyhy for supernumerary teeth location in pediatric dentistry. J Clin Pediatr Dent 2008 Sumer 32(4):273-6. 
26. Foster TD, Taylor GS. Characteristicsof supernumerery teeth in the upper central incisor region. Dent Pract Dent Rec 1969; 20: 8-12.

27.Tatlı U, Evlice B, Damlar İ, Arslanoğlu Z, Altan A. Çukurova bölgesinin süpernümerer diş karakteristikleri: çok merkezli retrospektif bir çalışma. Acta Odontol Turc 2014; 31(2): 84-8.

Yazışma Adresi:

Dr. Öğr. Üyesi Dilek MENZíLETOĞLU

Necmettin Erbakan Üniversitesi

Diş Hekimliği Fakültesi

Ağız, Diş ve Çene Cerrahisi AD

Karaciğan Mah Ankara Cad No:74

Karatay, Konya

Tel : : +90332 2200026

E-posta: dmenziletoglu@konya.edu.tr 